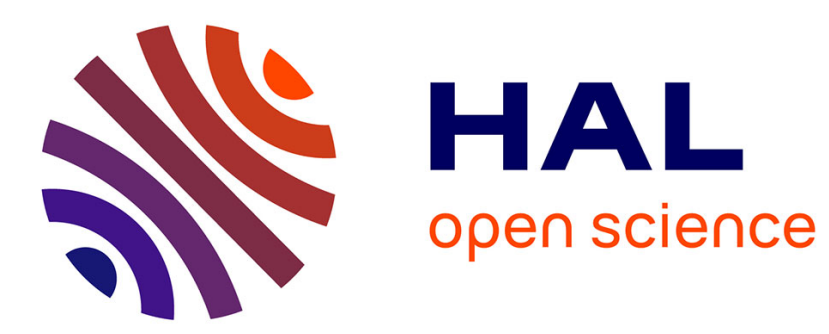

\title{
Energy transport of surface phonon polaritons propagating along a chain of spheroidal nanoparticles
}

Jose Ordonez-Miranda, Laurent Tranchant, Sergei Gluchko, Sebastian Volz

\section{To cite this version:}

Jose Ordonez-Miranda, Laurent Tranchant, Sergei Gluchko, Sebastian Volz. Energy transport of surface phonon polaritons propagating along a chain of spheroidal nanoparticles. Physical Review B: Condensed Matter and Materials Physics (1998-2015), 2015, 10.1103/PhysRevB.92.115409 . hal01357601

\section{HAL Id: hal-01357601 \\ https://hal.science/hal-01357601}

Submitted on 30 Aug 2016

HAL is a multi-disciplinary open access archive for the deposit and dissemination of scientific research documents, whether they are published or not. The documents may come from teaching and research institutions in France or abroad, or from public or private research centers.
L'archive ouverte pluridisciplinaire HAL, est destinée au dépôt et à la diffusion de documents scientifiques de niveau recherche, publiés ou non, émanant des établissements d'enseignement et de recherche français ou étrangers, des laboratoires publics ou privés. 


\title{
Energy transport of surface phonon polaritons propagating along a chain of spheroidal nanoparticles
}

\author{
Jose Ordonez-Miranda, ${ }^{1}$ Laurent Tranchant, ${ }^{2}$ Sergei Gluchko, ${ }^{1}$ and Sebastian Volz ${ }^{1, *}$ \\ ${ }^{1}$ Laboratoire EM2C, CNRS, CentraleSupélec, Université Paris-Saclay, Grande Voie des Vignes, \\ 92295 Chatenay-Malabry cedex, France \\ ${ }^{2}$ Department of Mechanical and Control Engineering, Kyushu Institute of Technology, 1-1 Sensui-cho, Tobata-ku, \\ Kitakyushu 804-8550, Japan
}

(Received 25 June 2015; revised manuscript received 23 August 2015; published 9 September 2015)

\begin{abstract}
We analyze in detail the energy transport of surface phonon polaritons propagating in a chain of spheroidal polar nanoparticles with both longitudinal and transversal polarizations. Explicit and closed-form expressions for the dispersion relation and propagation length are derived and used to determine the values of the nanoparticle polarizability and the interparticle distance that maximize the polariton propagation length. The thermal conductance in the ballistic regime and the thermal conductivity in the diffusive one are also determined and examined as a function of the geometry of the nanoparticles and their temperature. For a chain of cigar-shaped $\mathrm{SiC}$ nanoparticles in contact, an aspect ratio of 5, and surrounded by air; it is shown that: (i) The surface phonon polaritons propagate a distance of $10 \mu \mathrm{m}$ along a chain of 100 nanoparticles. This propagation length is one order of magnitude longer than that for spherical nanoparticles. (ii) The polariton thermal conductivity is comparable with the one of air in a wide range of temperatures and is $41 \mathrm{~mW} \mathrm{~m}^{-1} \mathrm{~K}^{-1}$ at $500 \mathrm{~K}$. (iii) The polariton thermal conductance increases with the temperature and at $500 \mathrm{~K}$ is $44 \mathrm{pW} \mathrm{K}^{-1}$, which represents $9 \%$ of the quantum of thermal conductance. In view of the ultralow phonon thermal conductivity of a chain of polar nanoparticles in contact and their high surface area-to-volume ratios, the proposed theoretical model and obtained results are expected to be useful to experimentally quantify the energy transport of surface phonon polaritons propagating along these nanostructures.
\end{abstract}

DOI: 10.1103/PhysRevB.92.115409

PACS number(s): 65.60.+a, 65.80.-g, 65.90.+i

\section{INTRODUCTION}

Since the initial development of the electromagnetic theory, it is known that when a small metallic particle is illuminated with light at a frequency tuned according to the resonant frequency of the negative real part of its permittivity, the electrons inside the particle oscillate cooperatively, generating a strong electrical field close to the particle surface [1]. At this resonant frequency, the particle behaves as a resonant cavity with strong absorption and scattering [2,3]. These electron resonances occur at visible and near-infrared frequencies, and they are nowadays called surface plasmon polaritons (SPPs). Surface phonon polaritons (SPhPs), on the other hand, stand for the phonon resonances in polar materials, and they are activated at midinfrared frequencies, mainly. Considering that the coupling among individual resonances of each nanoparticle may guide surface electromagnetic fields along a chain of nanoparticles, these structures have been proposed as potential waveguides of both SPPs and SPhPs. The propagation [4-18] and energy transport $[10,19]$ by SPPs propagating along chains of metallic nanoparticles have been widely investigated by many research groups, while analogous works dealing with SPhPs are scarce [20].

Based on the generalized Mie theory, Quinten et al. [4] found a propagation length of $909 \mathrm{~nm}$ for SPPs propagating along a chain of $\mathrm{Ag}$ spheres of $50 \mathrm{~nm}$ diameter separated by $75 \mathrm{~nm}$. This result was obtained for a chain with longitudinal polarization, which is expected to yield longer propagation lengths than the transversal one [5]. Taking into account the

\footnotetext{
*Corresponding author: sebastian.volz@ecp.fr
}

high-order multipole fields, Park and Stroud [7] calculated the SPP dispersion relation for a periodic chain of spherical metallic nanoparticles embedded in an isotropic host medium, and showed that the point-dipole approximation yields accurate results when the interparticle distance $d$ is equal to or greater than three times the particle radius $a(d \geqslant 3 a)$, provided that $d \ll \lambda$, where $\lambda$ is the light wavelength. This result was confirmed by Maier et al. [6], who used finite-difference time-domain simulations to analyze the SPP propagation along Au spherical nanoparticles with a radius of $25 \mathrm{~nm}$. The results of these two latter works were derived by using the Drule model to determine the permittivity of the metallic nanoparticles, and therefore they are not necessarily valid for polar or dielectric ones. More recently and under the point-dipole approximation, Alu and Engheta [9] developed closed-form analytical expressions for the dispersion relation of linear arrays of metamaterial/plasmonic nanoparticles, including the effect of material loss. Based on this dispersion relation, the conditions for minimal absorption and maximum bandwidth were also derived. Ben-Abdallah et al. [10] used the Landauer-Buttiker formalism and the kinetic theory to determine the plasmonic thermal conductance and the plasmonic thermal conductivity of a chain of copper nanoparticles, respectively. They found that the multipolar interactions among these nanoparticles increase significantly the ballistic thermal conductance, only when the permittivity of the host material is positive. In the diffusive regime, these latter authors showed that the plasmonic thermal conductivity of nanoparticle chains can reach $1 \%$ of the bulk Au thermal conductivity at $900 \mathrm{~K}$.

Surface phonon polaritons have been recently proposed as potential energy carriers to enhance significantly the phonon heat transport in polar nanofilms [21-28], nanowires 
[29], nanotubes [30], and gratings [31-33]. Various research groups have shown that these energy carriers have promising applications on the radiative heat transfer [34-37], highdensity infrared data storage [38], surface infrared absorption [39], coherent thermal emission [32], and photonics [40,41]. Despite these potential applications, the propagation and energy transport of SPhPs, analogous to those of SPPs, along chains of polar nanoparticles have not been explored to date. Taking into account the ultralow phonon thermal conductivity of a chain of polar nanoparticles, which exhibit high surface area-to-volume ratios, and the predominance of the surface effects at nanoscales, the SPhP energy transport along this structure is expected to dominate that of phonons, and to depend strongly on the size, shape, and separation of the nanoparticles.

In this paper, the transport properties and energy of SPhPs propagating along chains of spheroidal polar nanoparticles are analyzed in detail. This is done by including the material losses and by deriving closed-form analytical expressions for the dispersion relation and propagation length of SPhPs. The nanoparticle geometry and temperature dependences of the $\mathrm{SPhP}$ thermal conductance, in the ballistic regime, and of the SPhP thermal conductivity, in the diffusive regime, are determined for nanoparticles of $\mathrm{SiC}$. It is shown that both of these thermal properties increase with temperature and they can overcome their phonon counterparts. The proposed theoretical and practical framework provides a pathway to engineer thermally conductive polar materials for thermal management.

\section{SPhP DISPERSION RELATIONS}

Let us consider a linear chain of $N$ identical polar nanoparticles with relative permittivity $\varepsilon_{1}$, embedded in a dielectric medium of relative permittivity $\varepsilon_{2}$, as shown in Figs. 1(a) and 1(b). The radii of the spheroidal nanoparticles along the $z$ and $x$ axes are $a$ and $b$, respectively; and they are separated by a center-to-center distance $d$. When an external electrical field $\vec{E}_{\text {ext }}$ is applied parallel to the direction of the chain, the electrical dipoles of the polar molecules inside the polar nanoparticles align with $\vec{E}_{\text {ext }}$ and redistribute spatially around the nanoparticle surface mainly, as shown in Fig. 1(c). This surface polarization shows up parallel to the nanoparticle chain (longitudinal polarization) and induces a local electrical field $\vec{E}$ around each nanoparticle. On the other hand, when $\vec{E}_{\text {ext }}$ is perpendicular to the chain, the resulting surface polarization is also parallel to $\vec{E}_{\text {ext }}$ and induces the field $\vec{E}$ shown in Fig. 1(d). For both the longitudinal and transversal polarizations, the interactions among the electrical dipoles on the surface of the polar nanoparticles keep the propagation of SPhPs along the chain. These interactions strengthen as the distance between neighboring nanoparticles reduces, and therefore the highest SPhP energy transfer along the chain is expected to occur when the particles are in contact with each other $(d=2 a)$. The two polarization modes could also be activated by self-thermal emission $[32,42]$ or by the illuminated tip of an atomic force microscope [43]. Given that an arbitrary polarization can be expressed as a linear combination of the longitudinal and transversal ones $[9,44]$, the general problem of the SPhP propagation along nanoparticle chains can be split in these two orthogonal modes.

In general, the polarizable molecules of polar nanoparticles undergo multipole (dipole, quadrupole, octupole, etc.) interactions, which strengthen when the distance $d$ between nanoparticles of diameter $2 a$ reduces. This also applies for metallic nanoparticles supporting the propagation of plasmons [10], for which the energy contribution of these multipoles is proportional to $(d / 2 a)^{-(2 l+1)}$. This indicates that for $d>3 a$, the energy of the dipole interactions $(l=1)$ is much higher than those of all other higher-order interactions $(l=2,3, \ldots)$, and therefore, this condition $(d>3 a)$ on the interparticle distance represents a practical limit of application of the dipole approximation for plasmons. This was explicitly shown by Weber and Ford [8], and Ben-Adballah et al. [10] for chains of silver and copper nanoparticles, respectively. On the other hand, for metallic nanoparticles in touch $(d=2 a)$, the contribution to the energy transport of the higher-order multipoles can be higher than that of the dipole interactions. This result does not necessarily hold for polar nanoparticles, given that plasmons in metals show up at visible and near-infrared frequencies $[8,10]$, while SPhPs in polar materials appear at (lower) midinfrared ones [23]. The energy contribution of the dipole interactions in polar media at a given temperature could be higher than the corresponding one in metals, due to the frequency dependence of the Bose-Einstein distribution function involved in the quantification of the energy transport of both plasmons [10] and SPhPs [Eq. (24), below]. Thus, the dipole approximation is expected to yield more accurate results for polar nanoparticles than for metallic ones. This is the reason why, for the sake of simplicity and keeping analytical our approach, we are going to only consider the dipole interactions in this work. Taking into account that the multipole interactions in a chain with closely spaced nanoparticles can enhance the polariton thermal conductance rendered by the dipole interactions [10], our results for nanoparticles in contact represent the minimum (lower bound) for the energy transport of SPhPs.

To determine the dispersion relation of the SPhPs propagating along the nanoparticle chains shown in Figs. 1(a) and 1(b), we start calculating the electrical field $\vec{E}$ around a single polar nanoparticle and induced by its surface polarization. For a general polarization, this field is characterized by the dipole moment $\vec{p}$ of the nanoparticle and is given by [2]

$$
\vec{E}(\vec{r}, t)=\frac{1}{4 \pi \varepsilon_{2}}\left(\frac{\vec{A}}{r^{3}}-\frac{i k_{2} \vec{A}}{r^{2}}+\frac{\vec{B}}{r}\right) e^{i k_{2} r},
$$

where $\vec{r}$ is the position vector pointing from the dipole to the field point, the wave vector $k_{2}=\sqrt{\varepsilon_{2}} \omega / c, \omega$ is the excitation frequency, $c$ is the speed of light in vacuum, and

$$
\begin{aligned}
\vec{A} & =3 \hat{r}(\hat{r} \cdot \vec{p})-\vec{p}, \\
\vec{B} & =k_{2}^{2}[\vec{p}-\hat{r}(\hat{r} \cdot \vec{p})] .
\end{aligned}
$$

Note that in the quasistatic limit $\left(k_{2} r \ll 1\right)$, the dominant term of the electrical field $\vec{E}$ goes as $r^{-3}$, while the radiation component $\left(r^{-1}\right)$ dominates for $k_{2} r \gg 1$. For the longitudinal 


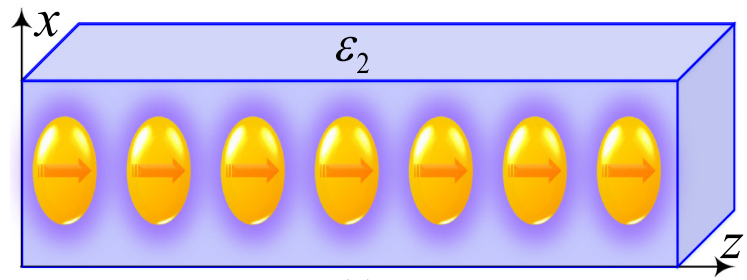

(a)

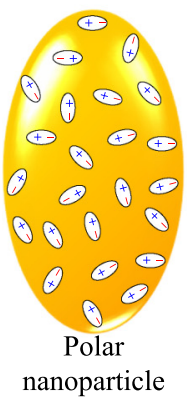

nanoparticle

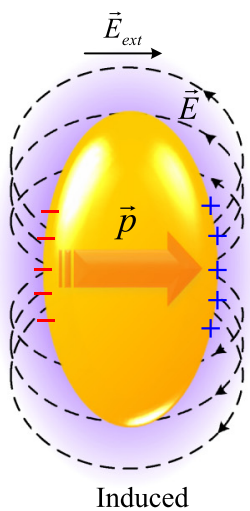

electrical field

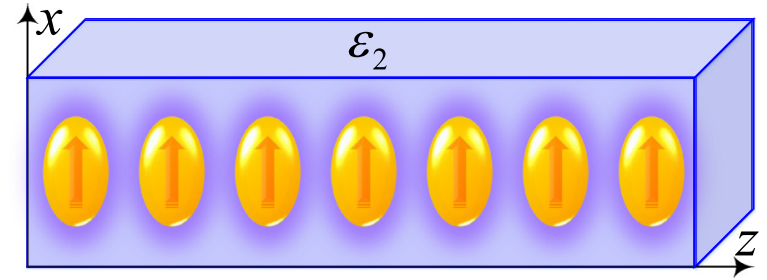

(b)

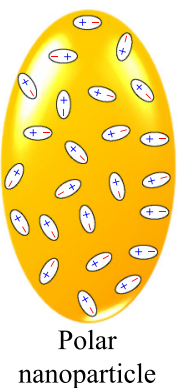

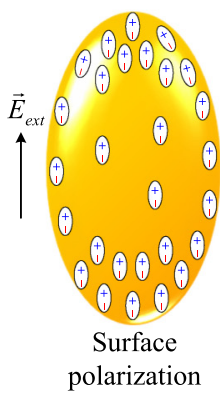

(d)

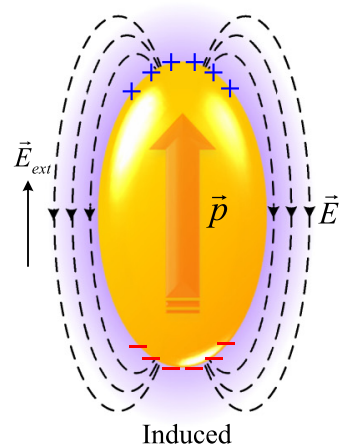

electrical field

FIG. 1. (Color online) Chain of spheroidal polar nanoparticles with (a) longitudinal and (b) transversal polarizations, which can be activated by an external electrical field $E_{\text {ext }}$, as shown in (c) and (d), respectively. The purple glow around the nanoparticles represents the coupled electromagnetic field induced by their surface polarization. The center-to-center distance between two neighboring spheroidal nanoparticles (ellipsoids with an axis of symmetry) is $d$ and their semiaxis lengths along the $x, y$, and $z$ axes are $b, a$, and $a$, respectively. Note that for both polarizations, the applied external field is parallel (antiparallel) to the dipole moment (one) induced by the polarization charge, inside the dielectric polar nanoparticle [2].

polarization shown in Fig. 1(c) $(\vec{p} \| \hat{r})$, Eqs. (2a) and (2b) reduce to $(\vec{A}, \vec{B})=(2 \vec{p}, 0)$, while for the transversal one shown in Fig. 1(d) $(\vec{p} \perp \hat{r})$, the vectors $\vec{A}$ and $\vec{B}$ take the form $(\vec{A}, \vec{B})=\left(-1, k_{2}^{2}\right) \vec{p}$. In these two polarizations, Eq. (1) yields the following dipole electrical field $\vec{E}$

$$
\vec{E}(\vec{r}, t)=\frac{\vec{p}}{4 \pi \varepsilon_{2}}\left(\frac{\gamma}{r^{3}}-\frac{i k_{2} \gamma}{r^{2}}+\frac{\delta k_{2}^{2}}{r}\right) e^{i k_{2} r},
$$

where $\gamma=2(\gamma=-1)$ and $\delta=0(\delta=1)$ for the longitudinal (transversal) polarization. For the linear chains of nanoparticles shown in Figs. 1(a) and 1(b), the field $\vec{E}_{n}$ at the $n^{\text {th }}$ dipole $\vec{p}_{n}$ is the sum of the fields due to all the other dipoles, and it is therefore given by

$$
\begin{aligned}
\vec{E}_{n}= & \frac{1}{4 \pi \varepsilon_{2}} \sum_{m \neq n} \vec{p}_{m}\left(\frac{\gamma}{|m-n|^{3} d^{3}}-\frac{i k_{2} \gamma}{|m-n|^{2} d^{2}}\right. \\
& \left.+\frac{\delta k_{2}^{2}}{|m-n| d}\right) e^{i k_{2}|m-n| d} .
\end{aligned}
$$

Taking into account that the SPhPs are propagating along the nanoparticle chain, the dipole moments can be considered as traveling waves $\vec{p}_{m}=\vec{p}_{0} e^{i \beta m d}$, with amplitude $\vec{p}_{0}$ and wave vector $\beta$. By inserting this relation into Eq. (4) and using the constitutive relation $\vec{p}_{n}=\alpha \vec{E}_{n}$, the following SPhP dispersion relation is obtained

$$
\begin{aligned}
\frac{2 \pi \varepsilon_{2}}{\alpha}= & \sum_{n=1}^{N} \frac{1}{(n d)^{3}}\left[\gamma\left(1-i k_{2} n d\right)+\delta\left(k_{2} n d\right)^{2}\right] \\
& \times e^{i k_{2} n d} \cos (\beta n d),
\end{aligned}
$$

where $\alpha$ is the nanoparticle electrical polarizability parallel to the corresponding dipole moment. According to Mie's theory $[3,45]$, the polarizability $\alpha$ of a small enough nanoparticle, such that $k a, k b \ll 1$, is given by

$$
\alpha^{-1}=\alpha_{s}^{-1}-\frac{i k_{2}^{3}}{6 \pi \varepsilon_{2}},
$$

where the second term is independent of the nanoparticle geometry and arises due to the radiation damping. On the other hand, the electrostatic component $\alpha_{s}$ depends on the nanoparticle size and shape, as follows [3]:

$$
\alpha_{s}=\frac{4 \pi a^{2} b}{3} \varepsilon_{2} \frac{\varepsilon_{1}-\varepsilon_{2}}{\varepsilon_{2}+L\left(\varepsilon_{1}-\varepsilon_{2}\right)},
$$

$L$ being the geometrical factor parallel to the dipole moment of the spheroidal nanoparticles $[3,46]$. This factor $L$ depends only on and increases with the aspect ratio $b / a$, such that it reduces to $L=1 / 3(L=1 / 2)$ for spherical (cylindrical) nanoparticles. According to Eqs. (6) and (7), the SPhP dispersion relation in Eq. (5) represents a relation between the nanoparticle geometry, in the left-hand side, and the propagation properties involved in the right-hand side, which rapidly decreases as the interparticle distance $d$ increases. The solution of Eq. (5) for the SPhP wave vector $\beta$ can be found by following a similar procedure than that developed for plasmonic spherical particles [9], and introducing the following three dimensionless parameters: $X=k_{2} d, \alpha_{e}=$ $\alpha_{s} k_{2}^{3} /\left(6 \pi \varepsilon_{2}\right)$, and $K=\beta / k_{2}$. In this way, the dispersion 
relation in Eq. (5) can be written as

$$
\begin{aligned}
-i+\alpha_{e}^{-1}= & \frac{3}{2 X^{3}}\left\{\gamma\left[f_{3}(K, X)-i x f_{2}(K, X)\right]\right. \\
& \left.+\delta X^{2} f_{1}(K, X)\right\},
\end{aligned}
$$

where $f_{j}(K, X)=L i_{j}\left(e^{i X(1+K)}\right)+L i_{j}\left(e^{i X(1-K)}\right), \quad L i_{j}(z)=$ $\sum_{n=1}^{\infty} z^{n} / n^{j}$ is the polylogarithm function of order $j$, which satisfies the following recurrence formula $L i_{j}(z)=$ $\int_{0}^{z} L i_{j-1}\left(z^{\prime}\right) d z^{\prime} / z^{\prime}$, with $L i_{1}(z)=-\ln (1-z)$ [47]. In deriving Eq. (8), we have assumed that the number of nanoparticles $N$ is large enough $(N X \gg 1)$ to replace the upper limit of the summation by infinite $(N \rightarrow \infty)$. Note that Eq. (8) allows us performing the analysis of the dispersion relation in terms of three dimensionless quantities: the normalized wave vector $K$, which is the unknown of the problem, the normalized interparticle distance $X$, and the normalized inverse polarizability $\alpha_{e}^{-1}$, related to the nanoparticle permittivity and geometry. We use this normalization to analytically determine some fundamental properties of the dispersion relation, without using its frequency dependence and therefore independently of the nanoparticle properties (contained in $\alpha_{e}^{-1}$ ) for a particular material.

The absorption of energy by real polar materials is taken into account by their complex permittivity $\varepsilon_{1}$, which turns the normalized polarizability $\alpha_{e}$ to a complex parameter. In the presence of this absorption, the surface electromagnetic waves under consideration are usually called Zenneck SPhPs [29], to distinguish them from those propagating along ideal materials without energy dissipation and in narrow intervals of frequency. For the Zenneck SPhPs considered in this work, Eq. (8) establishes that the SPhP wave vector can be written as $K=K_{R}+i K_{I}$, where its real $\left(K_{R}>0\right)$ and imaginary $\left(K_{I}>0\right)$ parts are associated with the propagation (along the $+z$ direction) and attenuation factor, respectively. For the practical cases of interest involving weak absorption and/or a small interparticle distance $d$, such that $K_{I} X \ll 1$, the exponentials involved in Eq. (8) can be well approximated by $e^{i X(1 \pm K)} \simeq\left(1 \mp K_{I} X\right) e^{i X\left(1 \pm K_{R}\right)}$, which along with the Taylor series expansion of the functions $f_{l}$, yields

$$
f_{j}(K, X)=f_{j}\left(K_{R}, X\right)+i K_{I} \frac{\partial f_{j}\left(K_{R}, X\right)}{\partial K_{R}} .
$$

Equation (9) allows us to factor $K_{I}$ out of $f_{j}(K, X)$ and therefore of the $\mathrm{SPhP}$ dispersion relation in Eq. (8). This result is valid as long as $K_{I} X \ll 1$, which, in terms of the $\mathrm{SPhP}$ propagation length $\Lambda=\left(2 \beta_{I}\right)^{-1}$ [23], implies that $\Lambda \gg d / 2$. This latter condition guarantees the SPhP propagation along many nanoparticles of the chain, as required. Given that the exponential factors $X\left(1 \pm K_{R}\right)$ are real numbers, the real and imaginary parts of the polylogarithm functions $L i_{j}\left(e^{i X\left(1 \pm K_{R}\right)}\right)$ involved in the definition of $f_{j}\left(K_{R}, X\right)$, can be separated and expressed in terms of the Clausens functions $C l_{j}(\theta)[9,47]$

$$
\begin{aligned}
& L i_{1}\left(e^{i \theta}\right)=C l_{1}(\theta)+\frac{i}{2}(\pi-\theta), \\
& L i_{2}\left(e^{i \theta}\right)=\frac{\pi^{2}}{6}-\frac{\theta}{4}(2 \pi-\theta)+i C l_{2}(\theta), \\
& L i_{3}\left(e^{i \theta}\right)=C l_{3}(\theta)+\frac{i}{12} \theta(\pi-\theta)(2 \pi-\theta),
\end{aligned}
$$

which hold for any $0 \leqslant \theta \leqslant 2 \pi$. The evaluation of the real functions $C l_{j}(\theta)$ in terms of summations or integrals is built in at well-known computing software as Mathematica and MATLAB, and they satisfy the recursive formula $\partial C l_{j}(\theta) / \partial \theta=$ $(-1)^{j} C l_{j-1}(\theta)$, with $C l_{1}(\theta)=-\ln [2 \sin (\theta / 2)]$. The periodicity of the cosine term in Eq. (5) or of the complex exponentials $e^{i X\left(1 \pm K_{R}\right)}$ in $f_{j}\left(K_{R}, X\right)$ establishes that the first (principal) period is given by $0<K_{R}<\pi / X$. Given that the existence and confinement of SPhPs to the surface of the nanoparticle chain is characterized by the difference $\left|\beta_{R}-k_{2}\right|\left(K_{R}=\beta_{R} / k_{2} \neq 1\right)$ of their wave vector $\left(\beta_{R}\right)$ with respect to that $\left(k_{2}\right)$ of light in the host medium, the condition $X=k_{2} d<\pi$ ensures that $K_{R}<1$ and $K_{R}>1$, and hence it guarantees the SPhP existence with long propagation lengths, due to the relatively small interparticle distances $d$ required to fulfill it. Taking into account that the dipole interactions keeping the SPhP propagation along the chain of nanoparticles are stronger for smaller interparticle distances, this period is the physically relevant one, for the wave vector $K_{R}$ (all higher-order periods reduce to this principal one, due to the periodicity). The condition $X<\pi\left(d<\lambda_{2} / 2=\pi / k_{2}\right)$ on the interparticle spacing and therefore on the particle size $\left(2 a \leqslant d<\lambda_{2} / 2\right)$ represents a fundamental condition for the existence of SPhPs propagating along the chain of nanoparticles. Equations (9) and (10) can now be used to extract the real and imaginary parts of the dispersion relation in Eq. (8).

\section{A. Short wave vector: $0<K_{R}<1$}

In this case, the SPhP wave vector is smaller than that of the light line $\left(\beta_{R}<k_{2}\right)$ and Eqs. (8) and (10) yield

$$
\begin{aligned}
& \operatorname{Re}\left(\alpha_{e}^{-1}\right)=\frac{3}{2 X^{3}} G\left(K_{R}, X\right)+\frac{3 \pi \gamma}{2 X} K_{R} K_{I}, \\
& \operatorname{Im}\left(\alpha_{e}^{-1}\right)=\frac{3 \pi}{4 X}\left[2 \delta+\gamma\left(1-K_{R}^{2}\right)\right]+\frac{3 K_{I}}{2 X^{3}} \frac{\partial G\left(K_{R}, X\right)}{\partial K_{R}},
\end{aligned}
$$

where the function $G$ is independent of $K_{I}$ and is given by

$$
G\left(K_{R}, X\right)=\gamma\left[g_{3}\left(K_{R}, X\right)+X g_{2}\left(K_{R}, X\right)\right]+\delta X^{2} g_{1}\left(K_{R}, X\right),
$$

$g_{j}\left(K_{R}, X\right)=C l_{j}\left[X\left(1+K_{R}\right)\right]+C l_{j}\left[X\left(1-K_{R}\right)\right]$.

The SPhP propagation is therefore driven by the Clausens functions $C l_{j}(\theta)$ of order $j=1,2,3$. For low frequencies and/or small interparticle distances, such that $X \ll 1$, the contributions of $g_{1}$ and $g_{2}$ disappear and $G\left(K_{R}, X\right) \rightarrow$ $\gamma g_{3}\left(K_{R}, X\right)$, for both polarizations. In absence of energy absorption $\left(K_{I}=0\right)$, Eqs. (11a) and (11b) reduce to the previous ones derived for ideal lossless materials [9], as expected. For real lossy materials $\left(K_{I}>0\right)$, on the other hand, Eqs. (11a) and (11b) are linear on $K_{I}$ and yield the following expressions for $K_{R}$ and $K_{I}$

$$
\begin{aligned}
\operatorname{Im}\left(\alpha_{e}^{-1}\right)= & \frac{3 \pi}{4 X}\left[2 \delta+\gamma\left(1-K_{R}^{2}\right)\right] \\
& +\frac{1}{\pi \gamma K_{R} X^{2}}\left(\operatorname{Re}\left(\alpha_{e}^{-1}\right)-\frac{3 G}{2 X^{3}}\right) \frac{\partial G}{\partial K_{R}},
\end{aligned}
$$




$$
K_{I}=\left\{\operatorname{Im}\left(\alpha_{e}^{-1}\right)-\frac{3 \pi}{4 X}\left[2 \delta+\gamma\left(1-K_{R}^{2}\right)\right]\right\} \frac{\partial K_{R}}{\partial \operatorname{Re}\left(\alpha_{e}^{-1}\right)} .
$$

For nanoparticles with a given polarizability $\alpha_{e}$, Eq. (13b) allows us to determine analytically $K_{I}$ after solving numerically Eq. (13a) for $K_{R}$. The propagation length $\Lambda=\left(2 \beta_{I}\right)^{-1}=$ $\left(2 k_{2} K_{I}\right)^{-1}$ can then be determined by means of

$$
\Lambda=\frac{1}{2} \frac{\partial \operatorname{Re}\left(\alpha_{e}^{-1}\right)}{\partial \omega} \frac{V_{g}}{\operatorname{Im}\left(\alpha_{e}^{-1}\right)-\frac{3 \pi}{4 X}\left[2 \delta+\gamma\left(1-K_{R}^{2}\right)\right]},
$$

where $V_{g}=\partial \omega / \partial \beta_{R}$ is the group velocity of the $\mathrm{SPhPs}$ propagating along the chain of nanoparticles. The direct proportionality of $V_{g}$ and $\Lambda$ indicates that faster SPhPs propagate larger distances.

\section{B. Long wave vector: $1<K_{R}<\pi / X$}

This is the case of SPhPs propagating with a wave vector greater than that of the light in the surrounding medium $\left(\beta_{R}>\right.$ $k_{2}$ ). under this condition, the dispersion relation in Eq. (8) splits into the following two equations

$$
\begin{aligned}
\operatorname{Re}\left(\alpha_{e}^{-1}\right) & =\frac{3 G}{2 X^{3}}, \\
K_{I} & =\operatorname{Im}\left(\alpha_{e}^{-1}\right) \frac{\partial K_{R}}{\partial \operatorname{Re}\left(\alpha_{e}^{-1}\right)},
\end{aligned}
$$

which are simpler than Eqs. (13a) and (13b) derived for short wave vectors. Equation (12a) shows that the real part of the $\mathrm{SPhP}$ wave vector $K$ (dispersion relation) is given by Eq. (15a), while its imaginary part in Eq. (15b) yields the following SPhP propagation length

$$
\Lambda=\frac{1}{2} \frac{\partial \operatorname{Re}\left(\alpha_{e}^{-1}\right)}{\partial \omega} \frac{V_{g}}{\operatorname{Im}\left(\alpha_{e}^{-1}\right)} .
$$

As in the previous case of short wave vectors, the proportionality between $\Lambda$ and $V_{g}$ still holds, which opens the possibility of transmitting SPhP energy at higher speeds for longer distances along the nanoparticle chain. In general, SPhPs can propagate with short $\left(0<K_{R}<1\right)$ and long $\left(1<K_{R}<\pi / X\right)$ wave vectors, but their guidance is stronger within this latter regime [9], which is going to be analyzed in further detail below.

\section{Longitudinal polarization (LP)}

In this section, we present the graphical analysis of Eqs. (15a) and (15b) for the LP $[(\gamma, \delta)=(2,0)]$ and in terms of the dimensionless parameters $X, \operatorname{Re}\left(\alpha_{e}^{-1}\right), \operatorname{Im}\left(\alpha_{e}^{-1}\right)$, and $K_{R}$. These normalized quantities depend on the frequency, but allow us to determine some general properties of the $\mathrm{SPhP}$ dispersion relation and propagation length, without involving their frequency dependence and independently of the nanoparticle properties for a particular material, as shown below.

The SPhP dispersion relation $\operatorname{Re}\left(\alpha_{e}^{-1}\right)$ vs $K_{R}$ and guidance region $\operatorname{Re}\left(\alpha_{e}^{-1}\right)$ vs $X$ are shown in Figs. 2(a) and 2(b), respectively. The monotonous decrease of $\operatorname{Re}\left(\alpha_{e}^{-1}\right)$ when $K_{R}$ increases implies that $\partial \operatorname{Re}\left(\alpha_{e}^{-1}\right) / \partial K_{R}<0$, which is valid for any distance $X$, as can be proved by taking the derivative of Eq. (15a) and using the properties of Clausen's functions. This fact along with the wave vector interval $1<K_{R}<\pi / X$ establish that the nanoparticle chain is able to support the propagation of SPhPs as long as the nanoparticle polarizability $\alpha_{e}$ satisfies the condition $3 G(\pi / X, X) / 2<X^{3} \operatorname{Re}\left(\alpha_{e}^{-1}\right)<$ $3 G(1, X) / 2$, which in terms of Clausen's functions reads

$$
\begin{aligned}
& 6\left[C l_{3}(X+\pi)+X C l_{2}(X+\pi)\right]<X^{3} \operatorname{Re}\left(\alpha_{e}^{-1}\right) \\
& \quad<3\left[C l_{3}(2 X)+X C l_{2}(2 X)+\xi(3)\right],
\end{aligned}
$$

where $\xi(3)=\mathrm{Cl}_{3}(2 \pi)$ is the Riemann $\zeta$ function. Note that when $X \rightarrow \pi\left(d=\lambda_{2} / 2\right)$, the interval of SPhP wave vectors and this region of guidance reduce to the single values $K_{R}=1$ $\left(\beta_{R}=k_{2}\right)$ and $\operatorname{Re}\left(\alpha_{e}^{-1}\right)=6 \pi^{-3} \xi(3)$, respectively. This indicates that at large interparticle distances $\left(d=\lambda_{2} / 2\right)$, the wave vector $K_{R}$ of the electromagnetic field propagating along the chain of nanoparticles is the same in presence and absence of the nanoparticles, which implies the disappearance of SPhPs. The presence and good guidance of SPhPs therefore require an interparticle spacing $d<\lambda_{2} / 2$, as was aforementioned. On the other hand, as $X \rightarrow 0\left(d \ll \lambda_{2} / 2 \pi\right)$ the guidance region
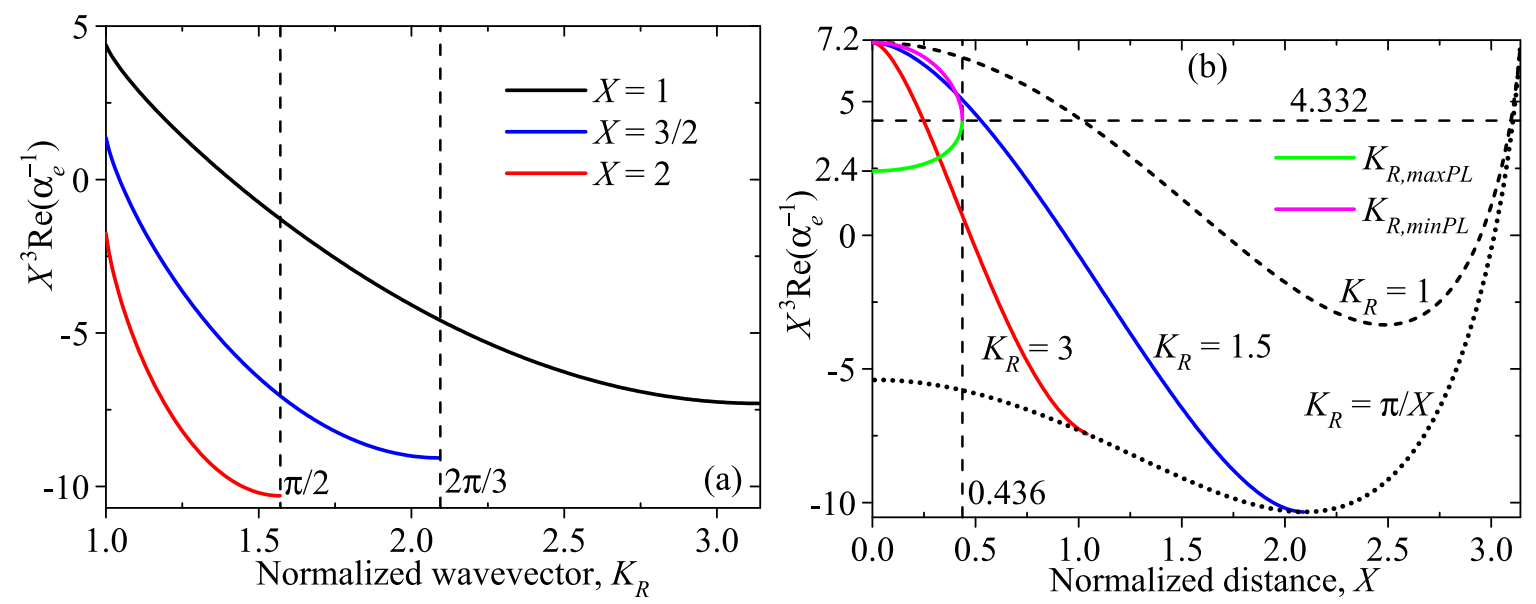

FIG. 2. (Color online) (a) Dispersion relation and (b) guidance region of SPhPs propagating along a chain of nanoparticles with longitudinal polarization. 
in Eq. (17) widens up and reaches its maximum amplitude in the interval $-5.41=-9 \xi(3) / 2 \leqslant X^{3} \operatorname{Re}\left(\alpha_{e}^{-1}\right) \leqslant 6 \xi(3)=$ 7.21. Therefore, in the long wavelength regime, the polarizability $\operatorname{Re}\left(\alpha_{e}^{-1}\right) \propto X^{-3}\left[\operatorname{Re}\left(\alpha_{s}^{-1}\right) \propto d^{-3}\right]$, which indicates that the $\mathrm{SPhP}$ propagation is driven by the relative volume (size) of the nanoparticles through the ratio $a^{2} b / d^{3}$ [see Eq. (7)]. These features of the SPhP guidance region are summarized in Fig. 2(b), and they also hold for a chain of lossless spherical nanoparticles, as shown by Alu and Engheta [9]. Furthermore, both Figs. 2(a) and 2(b) show that the reduction of $X$ and $\operatorname{Re}\left(\alpha_{e}^{-1}\right)$ increases the SPhP wave vector $K_{R}=\beta_{R} / k_{2}$ through values larger than that of the light in the host medium $\left(\beta_{R}>k_{2}\right)$. This increase is due to the permittivity mismatch between the nanoparticles and the host material, represents the strong presence of SPhPs, and establishes that their surface confinement and guidance along the chain of nanoparticles can be enhanced by tuning the interparticle distance and the real part of the inverse of the nanoparticle polarizability to low values.

Figure 3(a) shows the normalized propagation length $-\operatorname{Im}\left(\alpha_{e}^{-1}\right) k_{2} \Lambda$ as a function of the $\mathrm{SPhP}$ wave vector $K_{R}$ for different interparticle distances $X$. We have conveniently plotted the negative of $\operatorname{Im}\left(\alpha_{e}^{-1}\right) k_{2} \Lambda$ because of the negative values of $\operatorname{Im}\left(\alpha_{e}^{-1}\right)$, which is related to the negative wave vector derivative of $\operatorname{Re}\left(\alpha_{e}^{-1}\right)$ [Fig. 2(a)]. Note that for a given $K_{R}$, the propagation length increases as $X$ is scaled down, as expected. For $X<0.436$, the propagation length exhibits maximum and minimum values determined by $\partial \Lambda / \partial K_{R}=0$, which, according to Eqs. (15) and (16), reads

$$
\ln \left\{2\left[\cos (X)-\cos \left(X K_{R}\right)\right]\right\}+\frac{X \sin (X)}{\cos (X)-\cos \left(X K_{R}\right)}=0 .
$$

The solution of Eq. (18) yields the wave vector $K_{R}=K_{R \text {,opt }}$ determining the upper and lower bounds of the propagation length for the interparticle distances $(X<0.436)$ of interest. As displayed in Figs. 3(b) and 3(c), this optimum wave vector renders the longest (shortest) $\Lambda$ for $K_{R, \text { opt }}=K_{R, \operatorname{maxPL}}>$ $1.752\left(1<K_{R, \mathrm{opt}}=K_{R \text {, minPL }}<1.752\right)$, which is the interval of strong (weak) SPhP guiding. Note that the longest and shortest propagation lengths, as well as their difference, take longer values for shorter interparticle distances. According to Figs. 2(a), 2(b), and 3(c); the longest propagation length corresponds to a $\operatorname{Re}\left(\alpha_{e}^{-1}\right)$ smaller than the corresponding one to the shortest propagation length, which indicates that the SPhPs travel longer distances when they are more confined, as expected. It is therefore clear that in the LP, both the lateral confinement and the propagation length can be maximized by designing the chain of nanoparticles, such that $2.4<X^{3} \operatorname{Re}\left(\alpha_{e}^{-1}\right)<4.3$, with $X<0.436$ [Figs. 2(b) and $3(\mathrm{~b})]$.

\section{Transversal polarization (TP)}

The analyses of the dispersion relation and propagation length in terms of the normalized parameters involved in Eqs. (15a) and (15b) for the TP $[(\gamma, \delta)=(-1,1)]$ are graphically performed in this subsection. Given that $\alpha_{e}^{-1}$ depends on the frequency, its real part $\operatorname{Re}\left(\alpha_{e}^{-1}\right)$ is used to describe the dispersion relation.
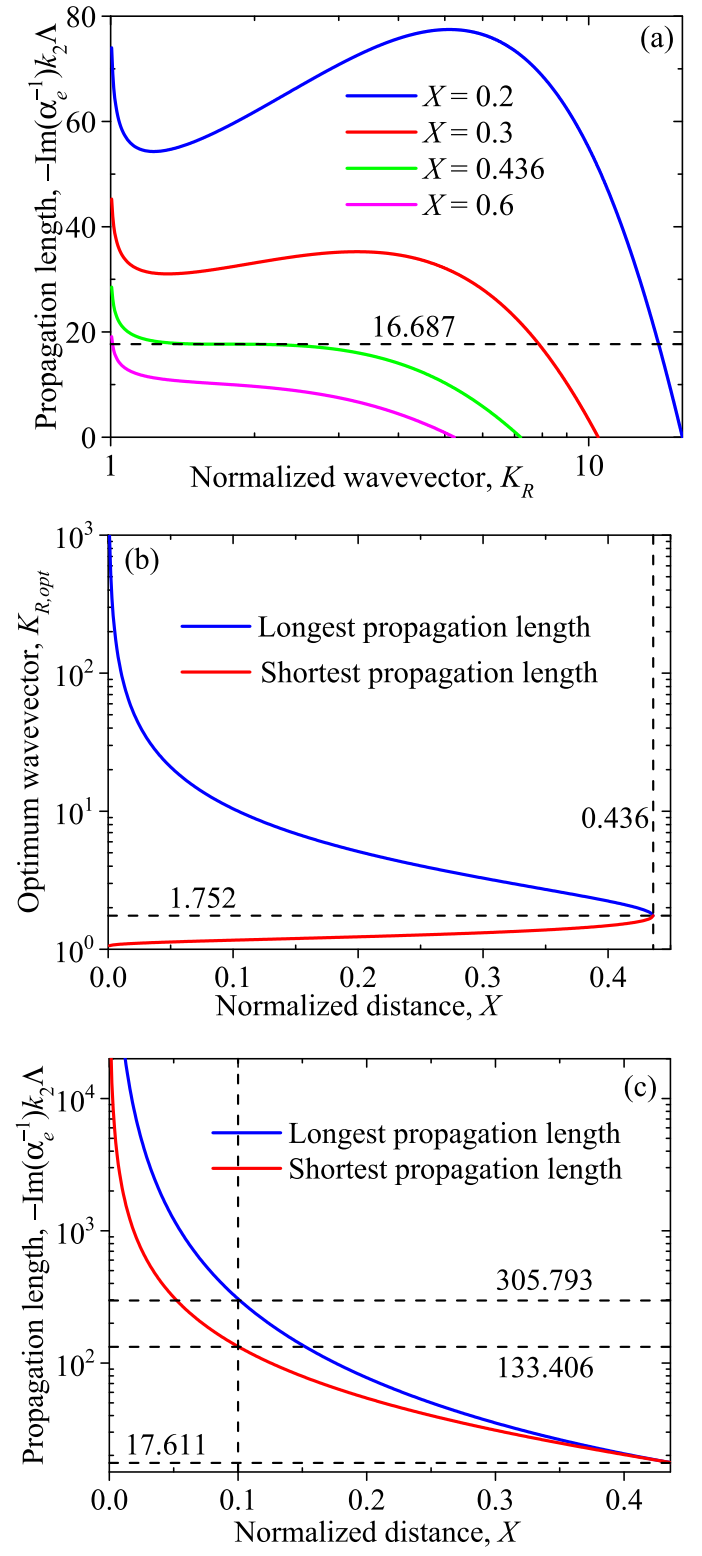

FIG. 3. (Color online) (a) Normalized propagation length as a function of the wave vector $K_{R}$, for different interparticle distances $X$. (b) Optimum wave vector to obtain the longest and shortest propagation lengths shown in (c), as a function of $X$. Calculations were done for the longitudinal polarization.

Figures 4(a) and 4(b) show the SPhP dispersion relation $\operatorname{Re}\left(\alpha_{e}^{-1}\right)$ vs $K_{R}$ and guidance region $\operatorname{Re}\left(\alpha_{e}^{-1}\right)$ vs $X$ of SPhPs propagating along a chain of particles with TP, respectively. Note that for $0<X<1.517$, contrary to the case of LP shown in Fig. 2(a), $\operatorname{Re}\left(\alpha_{e}^{-1}\right)$ exhibits a minimum at the wave vector $K_{R, \text { min }}$, which is given by $\partial \operatorname{Re}\left(\alpha_{e}^{-1}\right) / \partial K_{R}=0$. According to Eq. (15a) and the properties of Clausen's functions, this condition yields

$$
\begin{gathered}
C l_{2}\left(\theta_{+}\right)+C l_{2}\left(\theta_{-}\right)+X \ln \left(\frac{\sin \left(\theta_{+} / 2\right)}{\sin \left(\theta_{-} / 2\right)}\right) \\
=\frac{X^{2} \sin \left(X K_{R, \min }\right)}{\cos (X)-\cos \left(X K_{R, \min }\right)},
\end{gathered}
$$



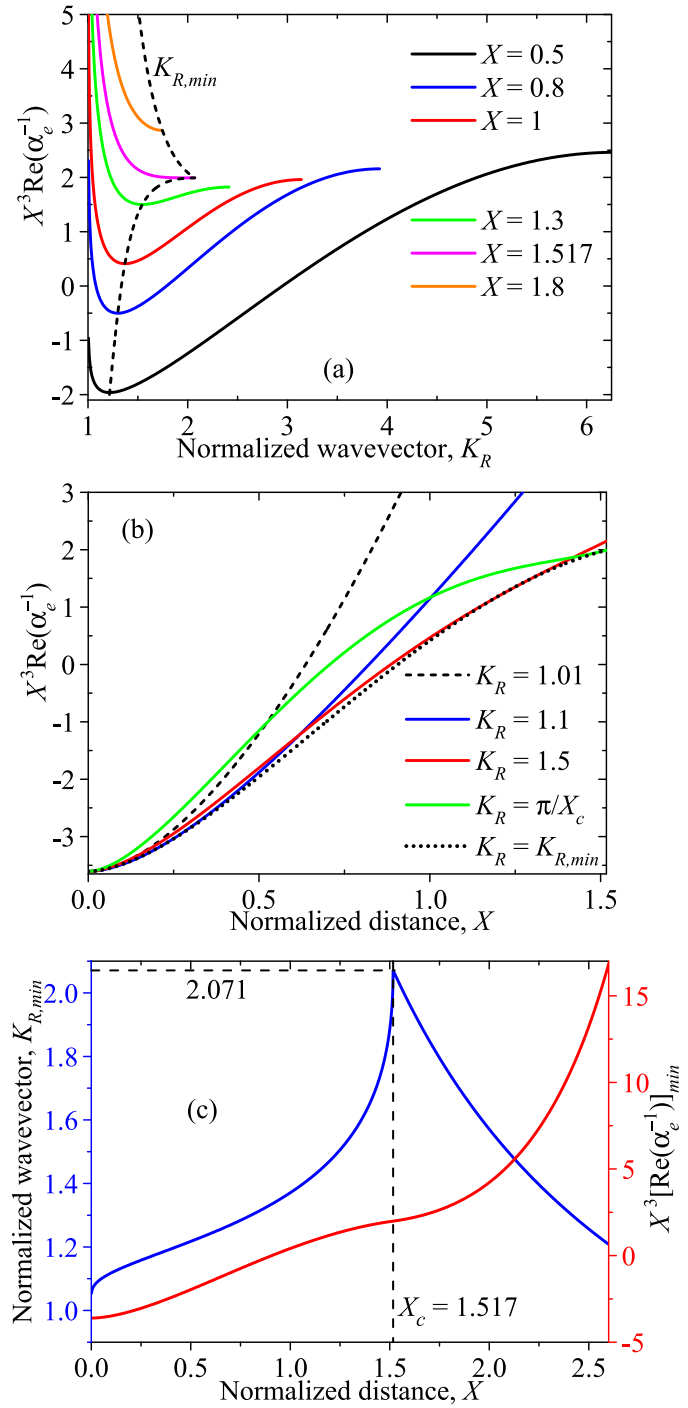

FIG. 4. (Color online) (a) Dispersion relation and (b) guidance region of SPhPs propagating along a chain of nanoparticles with transversal polarization. $\operatorname{Re}\left(\alpha_{e}^{-1}\right)$ reaches its minimum at the wave vector $K_{R, \min }$ shown in (c).

where $\theta_{ \pm}=X\left(K_{R, \min } \pm 1\right)$. The solution of Eq. (19) is plotted in Fig. 4(c), which shows that $K_{R \text {, min }}$ reaches its maximum at the critical thickness $X_{c}=1.517$. The values of $K_{R \text {, min }}$ for $X>X_{c}$ correspond to the lowest values of $\operatorname{Re}\left(\alpha_{e}^{-1}\right)$, as shown in Fig. 4(a). The mode with $K_{R}>$ $K_{R, \min }>1$ and hence with relatively strong $\mathrm{SPhP}$ confinement around the chain $\left(K_{R, \min }<K_{R}<\pi / X\right)$ is characterized by $\partial \operatorname{Re}\left(\alpha_{e}^{-1}\right) / \partial K_{R}>0$ and is defined within the bounded interval $3 G\left(K_{R, \min }, X\right) / 2<X^{3} \operatorname{Re}\left(\alpha_{e}^{-1}\right)<3 G(\pi / X, X) / 2$. By contrast, for $1<K_{R}<K_{R \text {, min }}, \partial \operatorname{Re}\left(\alpha_{e}^{-1}\right) / \partial K_{R}<0$ and $X^{3} \operatorname{Re}\left(\alpha_{e}^{-1}\right)>3 G\left(K_{R, \min }, X\right) / 2$. This latter condition on the nanoparticle polarizability $\alpha_{e}$ provides an infinitely wide spectrum of values for $\operatorname{Re}\left(\alpha_{e}^{-1}\right)$, but with $\mathrm{SPhP}$ wave vectors $\left(1<K_{R} \leqslant k_{R, \min } \leqslant 2.071\right)$ close to that of light inside the host medium (weak confinement). This is consistent with Eq. (7), which establishes that $\operatorname{Re}\left(\alpha_{e}^{-1}\right)$ takes higher values for smaller particles and/or particle materials similar to that of the host medium. The optimum design (strong SPhP confinement)

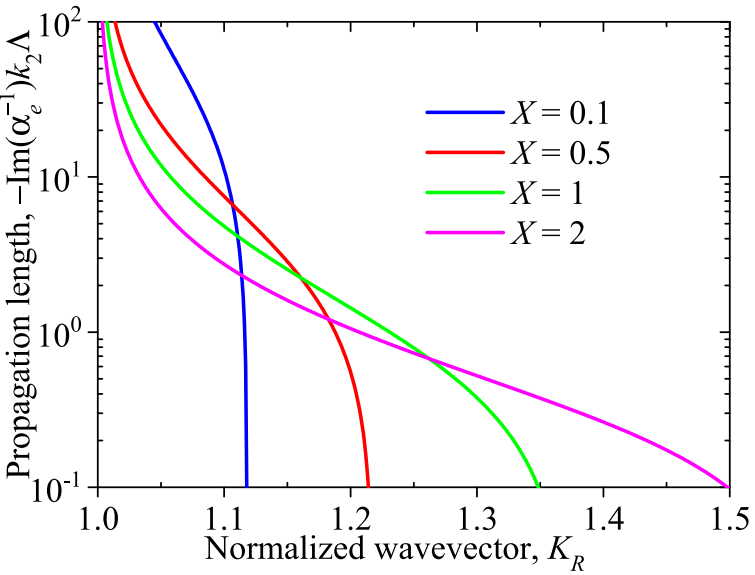

FIG. 5. (Color online) Normalized propagation length as a function of the wave vector $K_{R}$, for the transversal polarization and different interparticle distances $X$.

of the nanoparticle chain with TP should therefore involve a strong material (permittivity) mismatch between the host medium and the not-so-small nanoparticles. Furthermore, for interparticle distances $X<X_{c}$ and wave vectors $K_{R \text {, min }}<$ $K_{R}<\pi / X$ of major interest, the range of polarizabilities to support confined $\mathrm{SPhP}$ modes remains finite, as shown in Fig. 4(b).

The normalized propagation length $-\operatorname{Im}\left(\alpha_{e}^{-1}\right) k_{2} \Lambda$ as a function of the SPhP wave vector $K_{R}$ is shown in Fig. 5, for different interparticle distances $X$. Note that irrespective of $X$, the propagation length increases without limit as $K_{R}$ tends to the wave vector of light propagating in the host (nonabsorbing) medium, as expected. When the SPhP wave vector increases, the confinement strengthens but the propagation length remains small. A tradeoff between $K_{R}$ and $\Lambda$ is therefore required to obtain an optimum design of the chain of nanoparticles with TP.

\section{SPhP ENERGY TRANSPORT}

\section{A. Diffusive regime}

Assuming that the length of the nanoparticle chain is much longer than the SPhP propagation length, the SPhPs are expected to undergo many interactions with each other and therefore their heat transport can be described in the diffusive regime. This description is analogous to that of the Rosseland diffusion approximation [48] applied in radiative heat transfer and allows us to characterize the SPhP energy flux through a thermal conductivity. In this case, the heat flux $d q_{z}$ of SPhPs traveling at the position interval $d z$ during the time interval $d t$, along the nanoparticle chain of transversal section $S=\pi a b$ is given by

$$
d q_{z}=\frac{\hbar \omega f}{S} \frac{d z d p}{h d t},
$$

where $h=2 \pi \hbar$ is the Planck constant and $p=\hbar \beta_{R}$ is the momentum of SPhPs with a distribution function $f$ defined by the Boltzmann transport equation. Under the relaxation time approximation and steady-state heat transport along the chain, 
this equation yields

$$
f=f_{0}-\Lambda \frac{\partial f}{\partial z},
$$

where $f_{0}$ is the Bose-Einstein distribution function and $\Lambda$ is the SPhP propagation length (mean free path). Taking into account that the chain length is much longer than $\Lambda$, SPhP are in quasiequilibrium along the chain direction ( $z$ axis) and therefore we can use the diffusive approximation $\partial f / \partial z \approx$ $\partial f_{0} / \partial z$ [49]. Furthermore, by using the SPhP group velocity $V_{g}=d z / d t=\partial \omega / \partial \beta_{R}$, Eq. (21) takes the form

$$
d q_{z}=\frac{\hbar \omega}{2 \pi S}\left(f_{0}-\Lambda \frac{\partial f_{0}}{\partial z}\right) d \omega .
$$

Equation (22) yields the SPhP heat flux along the $+z$ direction and indicates that the one along the $-z$ direction can be determined by replacing $z$ by $-z$ in the spatial derivative of $f_{0}$. The net heat flux $Q_{z}$ along the $+z$ direction is then given by the integration over the frequency, as follows:

$$
Q_{z}=-\frac{1}{\pi S} \int \hbar \omega \Lambda \frac{\partial f_{0}}{\partial z} d \omega .
$$

Given that $f_{0}$ depends on the position $z$ through the temperature $T$ only, Eq. (23) establishes that in the diffusive regime, the SPhP heat transport is described by the Fourier's law with the following thermal conductivity

$$
\kappa=\frac{1}{\pi S} \int \hbar \omega \Lambda \frac{\partial f_{0}}{\partial T} d \omega .
$$

The lower and upper limits of integration in Eqs. (23) and (24) are defined by the frequency range of the dispersion relation that bounds the propagation of SPhPs with a propagation length $\Lambda$. The SPhP thermal conductivity $\kappa$ in Eq. (24) is half the value derived by Ben-Abdallah et al. [10] for metallic nanoparticle chains, by means of the kinetic theory. This discrepance could be due to a possible miscalculation of the density of states used in this theory.

\section{B. Ballistic regime}

In this regime, we assume that the $\mathrm{SPhP}$ propagation length is longer than the length of the nanoparticle chain, such that the SPhPs propagate along this chain without interacting each other. The SPhP energy transport can then be characterized by its thermal conductance, which is given by the Landauer formalism. Considering that the two extreme sides of the chain are perfectly connected to thermal baths, whose difference of temperature is much smaller than their average one $T$, the SPhP thermal conductance $G$ of the chain is given by $[10,29]$

$$
G=\frac{k_{B}^{2} T}{h}[J(A / T)-J(B / T)],
$$

where $k_{B}$ and $h=2 \pi \hbar$ are the Boltzmann and Planck constants, respectively; $A=\hbar \omega_{\min } / k_{B}$ and $B=\hbar \omega_{\max } / k_{B}$ are the respective minimum and maximum frequencies (in units of temperature) of the dispersion relation supporting the propagation of SPhPs; and

$$
J(x)=\frac{x^{2}}{e^{x}-1}-2 x \ln \left(1-e^{-x}\right)+2 \sum_{n=1}^{\infty} \frac{e^{-n x}}{n^{2}} .
$$

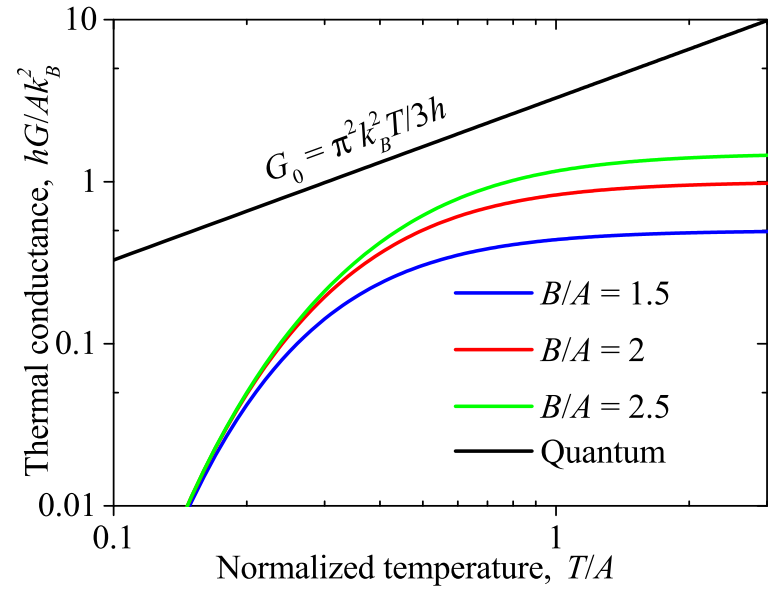

FIG. 6. (Color online) SPhP thermal conductance of a nanoparticle chain as a function of the normalized temperature $T / A$. The straight line stands for the SPhP quantum of thermal conductance $G_{0}$ of a polar nanowire.

Equations (25) and (26) were derived through the analytical integration of the integral involved in the Laundauer formula for the heat flux and assuming that the SPhPs are thermally generated by the hotter thermal bath, which excites and triggers the oscillations of the electrical dipoles of the polar nanoparticles. As a result of these oscillations, the nanoparticles emit an electrical field, which induces the excitation of neighboring electrical dipoles that keep the propagation of the field (SPhP) along the nanoparticle chain. Equation (25) establishes that in the ballistic regime, contrary to the diffusive one, the SPhP energy transport is independent of the propagation length and the particular characteristics of the dispersion relation, and is only determined by the relative values of the highest and lowest propagation frequencies with respect to the temperature. For midinfrared frequencies $\left(\omega_{\min }, \omega_{\max } \sim 10^{14} \mathrm{rad} / \mathrm{s}\right)$, where the $\mathrm{SPhP}$ resonances occur, the parameters $A, B \sim 764 \mathrm{~K}$, which indicates that $G$ depends strongly on the temperature, for temperatures comparable to room temperature $(T=300 \mathrm{~K})$. According to Fig. 6, the SPhP thermal conductance of the chain of nanoparticles increases with the temperature and the ratio $B / A$, but its values are smaller than the corresponding ones to the quantum of thermal conductance $G_{0}$, which holds for polar nanowires [29].

\section{RESULTS AND DISCUSSIONS}

The dispersion relation, propagation length, group velocity, thermal conductivity, and thermal conductance of SPhPs propagating along the nanoparticle chain shown in Fig. 1 are quantified in this section. This is done by means of the results derived in the previous Secs. II and III, and putting special emphasis on the effects of the nanoparticle geometry and temperature. Calculations are performed for nanoparticles of $\mathrm{SiC}$, which is an abundant crystalline polar material in nature, widely used in electronics [27,31,33], and whose complex permittivity $\varepsilon_{1}=\varepsilon_{R}+i \varepsilon_{I}$ is reported in the literature $[29,50]$ and shown in Fig. 7. The resonance peak of $\varepsilon_{I}$ occurs at 149 $\mathrm{Trad} / \mathrm{s}$, which indicates that the $\mathrm{SiC}$ absorbs more energy from the SPhPs at this frequency [2]. Furthermore, the real part 


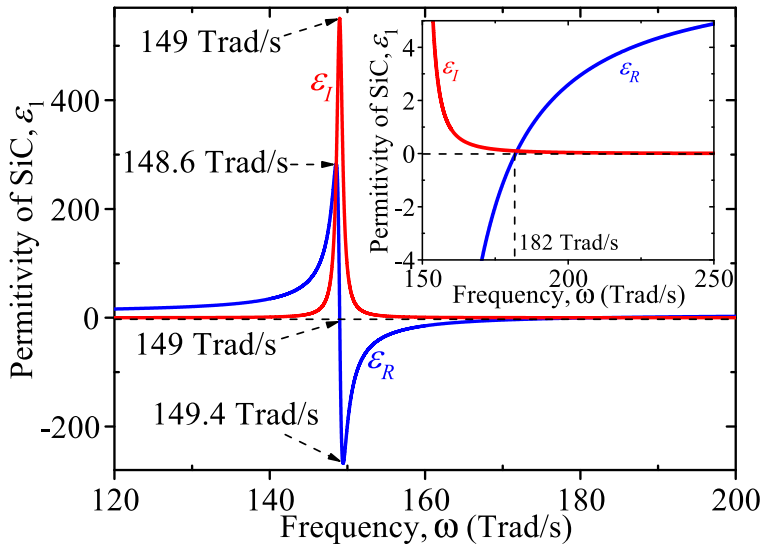

FIG. 7. (Color online) Real and imaginary parts of the relative permittivity $\varepsilon_{1}=\varepsilon_{R}+i \varepsilon_{I}$ of $\mathrm{SiC}$, as a function of frequency.

takes negative values $\left(\varepsilon_{R}<0\right)$ within the frequency interval $149 \mathrm{Trad} / \mathrm{s}<\omega<182 \mathrm{Trad} / \mathrm{s}$ that is expected to render the main contribution to the propagation and energy transport of SPhPs, as shown below. The values of the permittivity shown in Fig. 7 are valid for nanoparticle sizes larger than $5 \mathrm{~nm}$ [51]. Air is taken as a natural surrounded medium that is transparent $\left(\varepsilon_{2}=1\right)$ within the range of frequencies where the SiC supports the SPhP propagation. To determine the maximum possible energy that the SPhPs can transport along the chain, we have considered the minimum interparticle distance $d=2 a$, such that the spheroidal nanoparticles touch each other.

Figures 8(a), 8(b), and 8(c) show, respectively, the frequency dependence of the dispersion relation, propagation length, and group velocity of SPhPs propagating along a chain of $\mathrm{SiC}$ spheroidal nanoparticles with longitudinal polarization and five aspect ratios. For each nanoparticle geometry, the range of frequencies is determined by the real and positive solutions of Eqs. (8) and (16) for the propagation length. It is interesting to note that these frequencies are within the frequency interval $149 \mathrm{Trad} / \mathrm{s} \leqslant \omega \leqslant 182 \mathrm{Trad} / \mathrm{s}$, in which the real part of the permittivity of $\mathrm{SiC}$ is negative $\left[\operatorname{Re}\left(\varepsilon_{1}\right)<0\right]$ and therefore the excitation and propagation of SPhPs are expected to be strong $[23,28]$. As the frequency increases, the SPhP wave vector $\beta_{R}$ gets remarkably apart from the light line $\left(\beta_{R}=k_{2}\right)$, which enhances the confinement of the $\mathrm{SPhPs}$ to the nanoparticle surface, irrespective of their aspect ratio $b / a$. Note also that the amplitude of the frequency range supporting the SPhP propagation increases with $b / a$, such that for $b / a=5$, it nearly coincides with that in which $\operatorname{Re}\left(\varepsilon_{1}\right)<0$. The interception points of the curves indicates that there are certain frequencies at which the spherical $(b=a)$ or pancake-shaped $(b=a / 5)$ nanoparticles are able to support the same SPhP propagation than cigar-shaped ones ( $b=5 a)$, but with comparatively smaller propagation lengths, as shown in Fig. 7(b). The fact that $\Lambda$ increases with $b / a$ in the LP [Fig. 1(c)] is reasonable due to the reduction of the surface-to-surface distance among the nanoparticles, which strengthens the interactions among their surface dielectrical dipoles, when $b / a$ takes higher values. Spherical nanoparticles are therefore better than pancake-shaped ones, but less efficient than cigar-shaped ones for enhancing the SPhP propagation
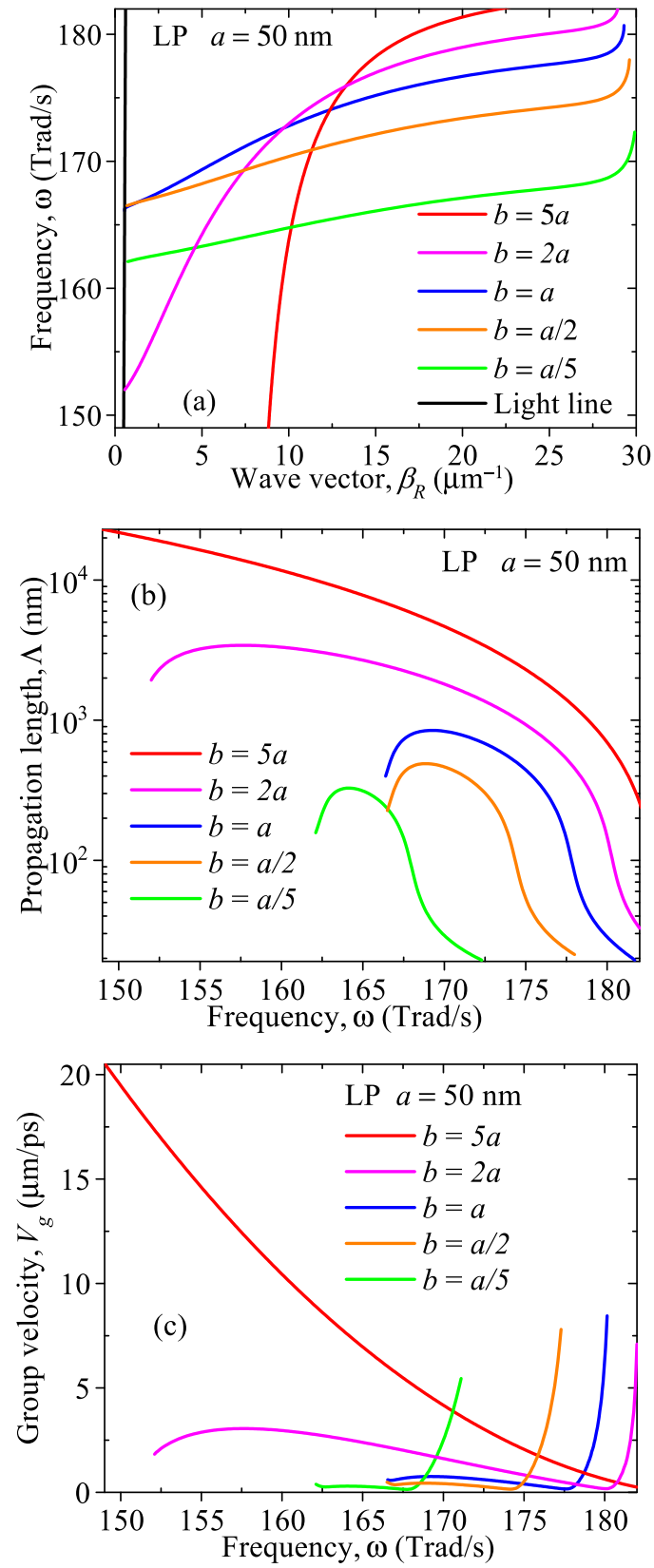

FIG. 8. (Color online) (a) Dispersion relation, (b) propagation length, and (c) group velocity of SPhPs propagating along a chain of $\mathrm{SiC}$ spheroidal nanoparticles with longitudinal polarization and five aspect ratios. Calculation were done for nanoparticles in contact $(d=2 a)$.

length. For $b=5 a=250 \mathrm{~nm}$ and $\Lambda=10^{4} \mathrm{~nm}$, the SPhPs are able to propagate along a chain of 100 nanoparticles, which is ten times the maximum number of spherical nanoparticles ( $b=a=50 \mathrm{~nm}$ ) that the SPhPs can travel along. According to Figs. 8(b) and 8(c), the SPhPs propagating along cigar-shaped nanoparticles travel longer distances at higher speeds, which occurs as the frequency reduces. By contrast, for spherical or pancake-shaped nanoparticles, the SPhP group velocity is comparatively small for most frequencies and increases sharply only at frequencies where the SPhP propagation length is negligible. This is why these latter frequencies are not of practical interest. 

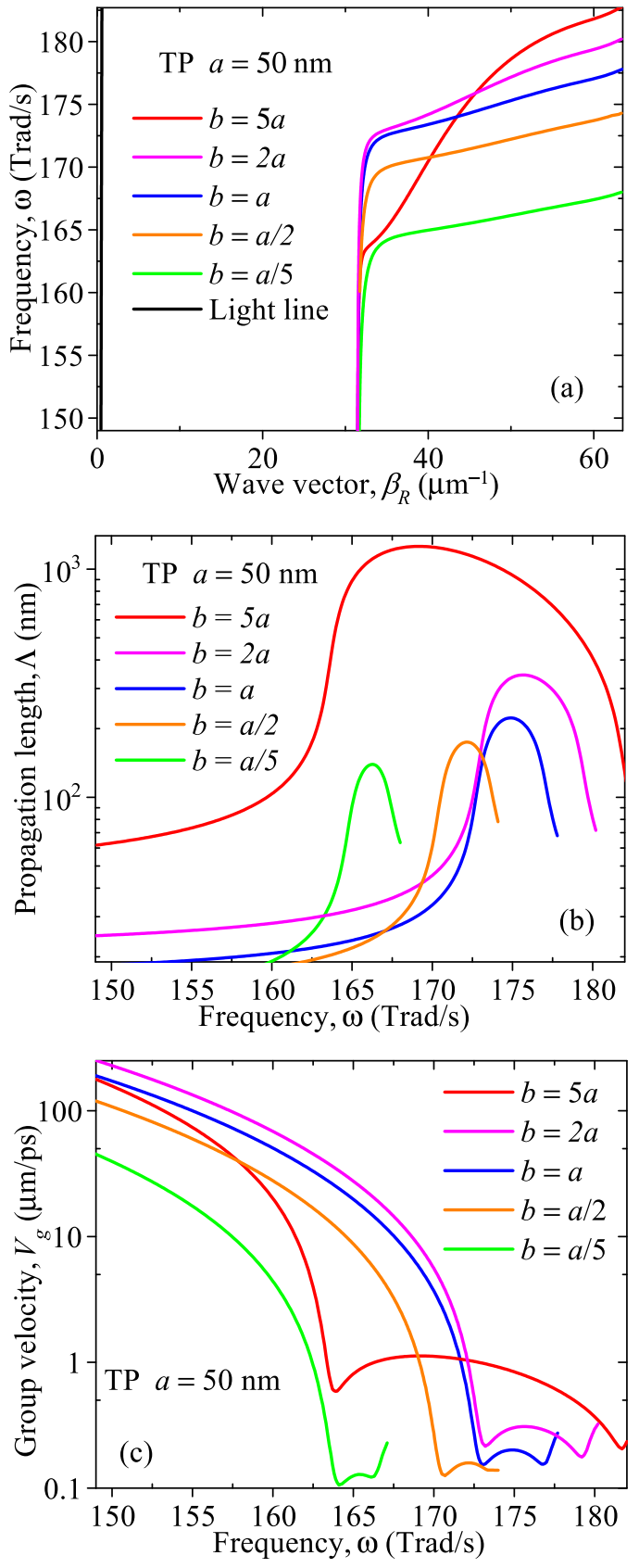

FIG. 9. (Color online) (a) Dispersion relation, (b) propagation length, and (c) group velocity of SPhPs propagating along a chain of $\mathrm{SiC}$ spheroidal nanoparticles with transversal polarization and five aspect ratios. Calculation were done for nanoparticles in contact $(d=2 a)$.

The dispersion relation, propagation length, and group velocity of SPhPs propagating along a chain of $\mathrm{SiC}$ spheroidal nanoparticles with transversal polarization are shown in Figs. 9(a), 9(b), and 9(c); as a function of the frequency, respectively. As in the case of LP, the amplitude of the frequency interval for which the SPhPs propagate, increases with the aspect ratio $b / a$ of the nanoparticles. Note that for low frequencies $\omega<165 \mathrm{Trad} / \mathrm{s}$, the $\mathrm{SPhP}$ wave vector $\beta_{R}$ is practically parallel to the light line and independent of the nanoparticle aspect ratio $b / a . \beta_{R}$ and hence the
SPhP confinement increases for higher frequencies, which coincides with the behavior of the dispersion relation shown in Fig. 8(a), for the LP. On the other hand, the maximum values of the propagation length increases with $b / a$, which also determines the frequencies at which they occur. For cigar-shaped nanoparticles $(b=5 a)$, the peak of $\Lambda \approx 10^{3} \mathrm{~nm}$ is comparatively much higher than those for spherical and pancake-shaped ones, and it supports the propagation of SPhPs along 10 nanoparticles. This is one order of magnitude smaller than the number of nanoparticles that the SPhPs can propagate along a chain with nanoparticles of the same size and shape, but with LP. This is expected, given that the major density of the nanoparticle electrical dipoles in the TP is located in the poles of each nanoparticle, which does not favor the interactions among them, as it occurs in the LP. Given that the maximum propagation lengths for $b=a$ and $b=a / 5$ are only of the order of the diameter $2 a$ of the nanoparticles, the propagation of the SPhPs along a chain of these nanoparticles is not possible in the TP. Furthermore, the enhancement of the propagation length and the amplitude of the frequency range with the ratio $b / a$, indicates that for both the LP and TP, cigar-shaped nanoparticles render SPhPs for longer distances and wider ranges of frequency than the usual spherical ones. For $b=5 a$ and within the frequency interval of major interest, in which the confinement and propagation length are relatively high, the $\mathrm{SPhPs}$ propagate with a speed of about $1 \mu \mathrm{m} / \mathrm{ps}$, as shown in Fig. 9(c). These SPhPs can therefore travel along a chain of 100 nanoparticles in about 10 ps.

Figures 10(a) and 10(b) show respectively the frequency spectrum and the temperature dependence of the SPhP thermal conductivity $\kappa$ of a chain of nanoparticles with LP. Note that for each aspect ratio $b / a$, the spectrum of $\kappa$ follows a similar frequency behavior than the propagation length $\Lambda$ shown in Fig. 8(b). This is consistent with Eq. (24) and confirms the ability of cigar-shaped nanoparticles for enhancing the energy transport of SPhPs. Even though the spectra for spherical and pancake-shaped nanoparticles are associate with relatively small propagation lengths [Fig. 8(b)], at certain frequencies they overlap with the spectrum for cigar-shaped ones due to its inverse dependence on the nanoparticle cross section area $S=\pi a b$. Based on Figs. 8(b) and 10(a), the SPhP propagation along pancake-shaped nanoparticles appears in a narrower frequency interval and lower propagation lengths than those for spherical ones, but its spectrum of thermal conductivity is comparatively higher, due to the fact that it occurs at relatively lower frequencies, as established by the Bose-Einstein distribution function involved in Eq. (24). As a result of this behavior, the SPhP thermal conductivity (area under the spectrum curves) of the chain with pancake-shaped nanoparticles $(b=a / 5)$ is a little higher than the one with spherical nanoparticles $(b=a)$, as shown in Fig. 10(b). The SPhP thermal conductivity for $b=5 a$ increases with temperature and is one order of magnitude higher than that for the other two geometries and comparable to the one of air. This relatively sizable increase of $\kappa$ for $b=5 a$ is generated by the strengthening of the surface dipole interactions as the aspect ratio $b / a$ of the nanoparticles increases. This indicates that cigar-shaped nanoparticles with LP and aspect ratios $b / a \gtrsim 5$ are the suitable candidates to observe a sizable energy transport by SPhPs. 


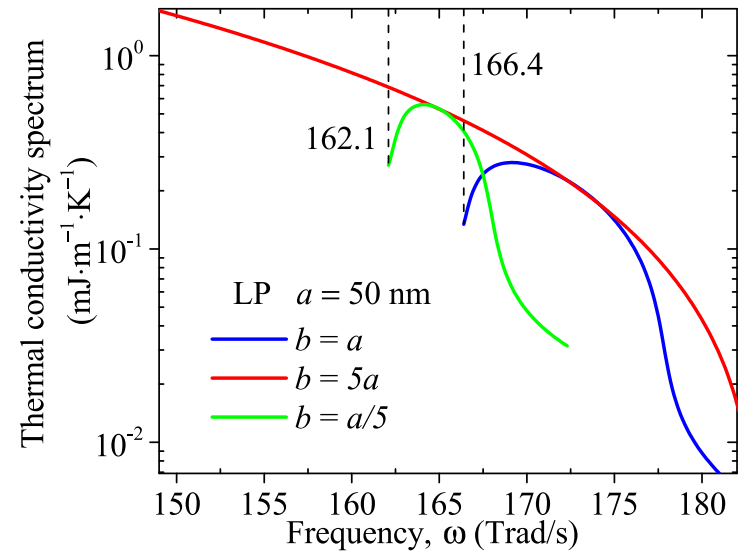

(a)

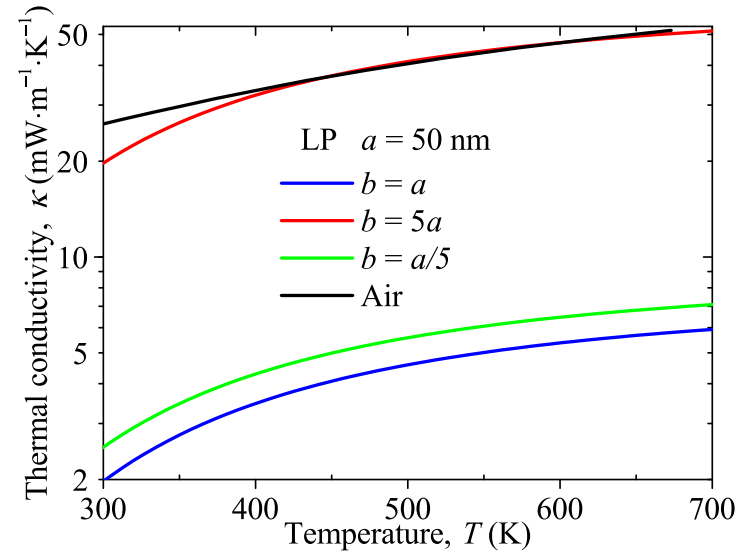

(b)

FIG. 10. (Color online) (a) Frequency spectrum and (b) temperature dependence of the SPhP thermal conductivity of a chain of SiC nanoparticles with longitudinal polarization and three aspect ratios. Calculation were done for nanoparticles in contact ( $d=2 a)$, and in (a) we used $T=500 \mathrm{~K}$.

The frequency spectrum and temperature dependence of the SPhP thermal conductivity $\kappa$ of a chain of nanoparticles with TP are shown in Figs. 11(a) and 11(b), respectively. As in the case of LP, the behavior of the $\kappa$ spectra is dictated by the propagation length shown in Fig. 9(b), but their magnitudes are determined by the frequency at which they occur. The peak of the $\kappa$ spectrum for $b=a / 5$ is higher than the ones for the other two nanoparticle geometries because of its presence at a comparatively lower frequency. This is why the SPhP thermal conductivity of a chain with these pancake-shaped nanoparticles is much higher than those for spherical and cigar-shaped ones. This is opposite to the result for LP but reasonable given that in the TP, the reduction of $b$ keeping constant $a$, enhances the interaction between the electrical dipoles placed at the north and south poles of the nanoparticles, which results in a major energy transport by SPhPs. However, the SPhP thermal conductivity in the TP is comparatively much smaller than that for the LP shown in Fig. 10(b), which ratifies that the SPhP energy transport along the chain of polar nanoparticles is dominated by this latter polarization.

Figure 12 shows the SPhP thermal conductance $G$ of a nanoparticle chain with LP, as a function of the temperature. Given that the amplitude of the frequency interval supporting the propagation of SPhPs increases with $b / a$, and $G$ depends only on the minimum and maximum frequencies of this range [Eq. (25)], the thermal conductance is higher for chains with cigar-shaped nanoparticles, as is the case of the corresponding thermal conductivity in the diffusive regime. The thermal conductance increases with the temperature, such that when the chain temperature rises from $300 \mathrm{~K}$ to $700 \mathrm{~K}, G$ increases by $175 \%$, for $b / a=5$. This enhancement is higher for spherical $(200 \%)$ and pancake-shaped $(187 \%)$ nanoparticles, but through smaller thermal conductances. The energy transport by SPhPs in the ballistic regime can therefore be enhanced by means of lengthened nanoparticles $(b>a)$ in the direction perpendicular to the chain one. This trend holds for both the LP and TP, given that the corresponding ranges of

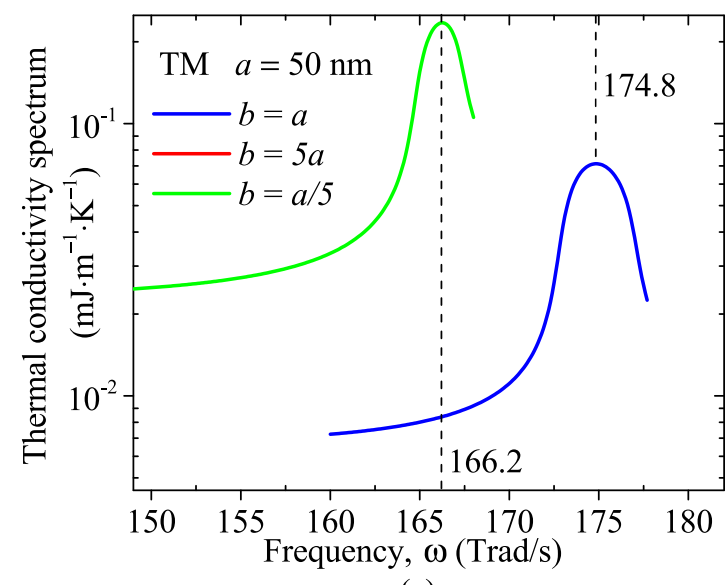

(a)

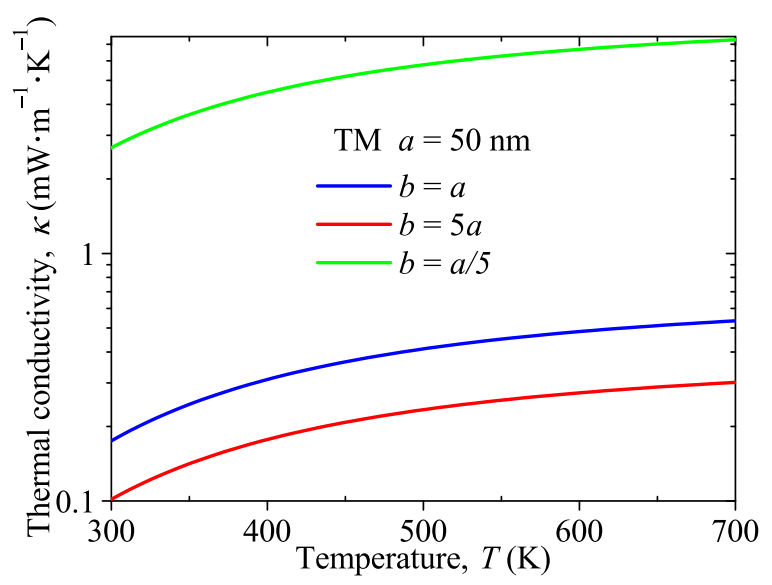

(b)

FIG. 11. (Color online) (a) Frequency spectrum and (b) temperature dependence of the SPhP thermal conductivity of a chain of SiC nanoparticles with transversal polarization and three aspect ratios. Calculation were done for nanoparticles in contact $(d=2 a)$, and in (a) we used $T=500 \mathrm{~K}$. 


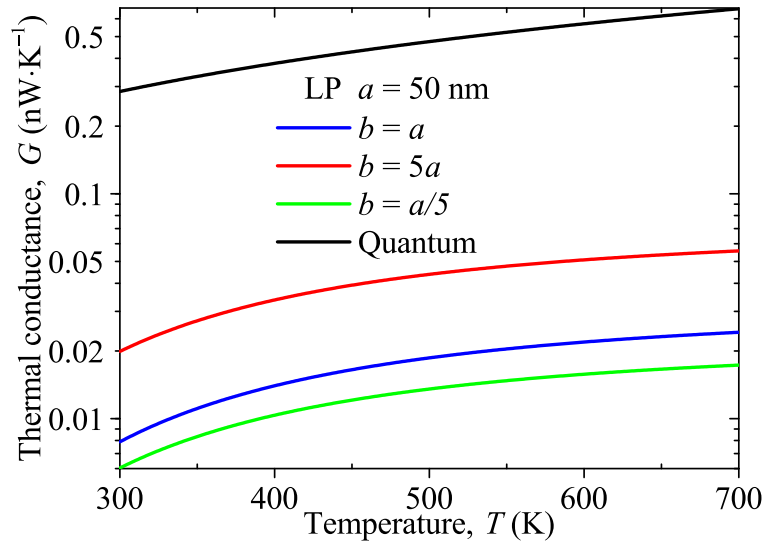

FIG. 12. (Color online) Temperature dependence of the SPhP thermal conductance of a nanoparticle chain with longitudinal polarization. The black line corresponds to the quantum of thermal conductance $G_{0}=\pi^{2} k_{B}^{2} T / 3 h$ and the calculation were done for nanoparticles in contact $(d=2 a)$.

frequency supporting the $\mathrm{SPhP}$ propagation are practically the same, as shown in Figs. 8 and 9.

\section{CONCLUSIONS}

The energy transport of surface phonon polaritons propagating along a chain of spheroidal polar nanoparticles has been analyzed in detail, for both the longitudinal and transversal polarizations. Under the dipolar approximation for each nanoparticle, explicit and closed-form expressions for the dispersion relation and propagation length have been derived and used to determine the values of the nanoparticle polarizability and the interparticle distance that optimize the polariton propagation length. The thermal conductance and thermal conductivity of polaritons have been also determined and examined as a function of the geometry of the nanoparticles and their temperature. For a chain of $\mathrm{SiC}$ nanoparticles with an aspect ratio of 5, longitudinal polarization, and surrounded by air; it has been shown that cigar-shaped nanoparticles support the propagation of surface phonon polaritons in wider ranges of frequency, with longer propagation lengths, and higher thermal conductivities than spherical ones. The polariton thermal conductivity of this chain at $500 \mathrm{~K}$, is $41 \mathrm{~mW} \mathrm{~m}^{-1} \mathrm{~K}^{-1}$, which is comparable to that of air and ten times higher than the corresponding one in the transversal configuration. Furthermore, the polariton thermal conductance is not so sensitive to the chain polarization, increases with the temperature, and at $500 \mathrm{~K}$ is $44 \mathrm{pW} \mathrm{K}^{-1}$, which represents $9 \%$ of the quantum of thermal conductance. The proposed theoretical formalism and obtained results are expected to be useful for guiding the design and fabrication of chains of nanoparticles with optimized energy transport by surface phonon polaritons.
[1] M. Kerker, Appl. Opt. 30, 4699 (1991).

[2] J. D. Jackson, Classical electrodynamics (Wiley, New York, 1998).

[3] C. F. Bohren and D. R. Huffman, Absorption and Scattering of Light by Small Particles (Wiley, New York, 1983).

[4] M. Quinten, J. R. Leitner, and F. R. Aussenegg, Opt. Lett. 23, 1331 (1998).

[5] M. L. Brongersma, J. W. Hartman, and H. A. Atwater, Phys. Rev. B 62, R16356 (2000).

[6] S. A. Maier, P. G. Kik, and H. A. Atwater, Phys. Rev. B 67, 205402 (2003).

[7] S. Y. Park and D. Stroud, Phys. Rev. B 69, 125418 (2004).

[8] W. H. Weber and G. W. Ford, Phys. Rev. B 70, 125429 (2004).

[9] A. Alu and N. Engheta, Phys. Rev. B 74, 205436 (2006).

[10] P. Ben-Abdallah, K. Joulain, J. Drevillon, and C. Le Goff, Phys. Rev. B 77, 075417 (2008).

[11] K. B. Crozier, E. Togan, E. Simsek, and T. Yang, Opt. Express 15, 17482 (2007).

[12] R. A. Bustos-Marun, E. A. Coronado, and H. M. Pastawski, Phys. Rev. B 82, 035434 (2010).

[13] I. B. Udagedara, I. D. Rukhlenko, and M. Premaratne, Phys. Rev. B 83, 115451 (2011).

[14] A. Alu and N. Engheta, Phys. Rev. B 78, 085112 (2008).

[15] P. J. Compaijen, V. A. Malyshev, and J. Knoester, Phys. Rev. B 87, 205437 (2013).

[16] C. R. Simovski, A. J. Viitanen, and S. A. Tretyakov, Phys. Rev. E 72, 066606 (2005).
[17] T. J. Antosiewicz, S. P. Apell, I. Zach, M. Zoric, and C. Langhammer, Appl. Phys. Lett. 109, 247401 (2012).

[18] I. L. Rasskazov, S. V. Karpov, and V. A. Markel, Phys. Rev. B 90, 075405 (2014).

[19] P. Ben-Abdallah, R. Messina, S. A. Biehs, M. Tschikin, K. Joulain, and C. Henkel, Phys. Rev. Lett. 111, 174301 (2013).

[20] R. Arias and D. L. Mills, Phys. Rev. B 68, 245420 (2003).

[21] Dye-Zone A. Chen and G. Chen, Appl. Phys. Lett. 91, 121906 (2007).

[22] Dye-Zone A. Chen and G. Chen, Front. Heat Mass Transfer 1, 023005 (2010).

[23] J. Ordonez-Miranda, L. Tranchant, T. Tokunaga, B. Kim, B. Palpant, Y. Chalopin, T. Antoni, and S. Volz, J. Appl. Phys. 113, 084311 (2013).

[24] J. Ordonez-Miranda, L. Tranchant, B. Kim, Y. Chalopin, T. Antoni, and S. Volz, Appl. Phys. Express 7, 035201 (2014).

[25] J. Ordonez-Miranda, L. Tranchant, T. Antoni, Y. Chalopin, and S. Volz, J. Appl. Phys. 115, 054311 (2014).

[26] P. B. Catrysse and S. H. Fan, Phys. Rev. B 75, 075422 (2007).

[27] F. Marquier, K. Joulain, and J. J. Greffet, Opt. Lett. 29, 2178 (2004).

[28] Dye-Zone A. Chen, A. Narayanaswamy, and G. Chen, Phys. Rev. B 72, 155435 (2005).

[29] J. Ordonez-Miranda, L. Tranchant, B. Kim, Y. Chalopin, T. Antoni, and S. Volz, Phys. Rev. Lett. 112, 055901 (2014).

[30] X. J. G. Xu, B. G. Ghamsari, J. H. Jiang, L. Gilburd, G. O. Andreev, C. Y. Zhi, Y. Bando, D. Golberg, P. Berini, and G. C. Walker, Nature Commun. 5, 4782 (2014). 
[31] H. J. Bakker, S. Hunsche, and H. Kurz, Rev. Mod. Phys. 70, 523 (1998).

[32] J. J. Greffet, R. Carminati, J. P. Joulain, K. Mulet, S. Mainguy, and Y. Chen, Nature (London) 416, 61 (2002).

[33] J. Le Gall, M. Olivier, and J. J. Greffet, Phys. Rev. B 55, 10105 (1997).

[34] M. Francoeur, M. P. Menguc, and R. Vaillon, Appl. Phys. Lett. 93, 043109 (2008).

[35] K. Torii, M. Ono, T. Sota, T. Azuhata, S. F. Chichibu, and S. Nakamura, Phys. Rev. B 62, 10861 (2000).

[36] P. O. Chapuis, M. Laroche, S. Volz, and J. J. Greffet, Appl. Phys. Lett. 92, 201906 (2008).

[37] P. O. Chapuis, S. Volz, C. Henkel, K. Joulain, and J. J. Greffet, Phys. Rev. B 77, 035431 (2008).

[38] N. Ocelic and R. Hillenbrand, Nat Mater. 3, 606 (2004).

[39] H. C. Kim and X. Cheng, Opt. Lett. 35, 3748 (2010).

[40] T. Taubner, F. Keilmann, and R. Hillenbrand, Nano Lett. 4, 1669 (2004).

[41] E. Rousseau, A. Siria, G. Jourdan, S. Volz, F. Comin, J. Chevrier, and J. J. Greffet, Nature Photon 3, 514 (2009).

[42] A. Babuty, K. Joulain, P. O. Chapuis, J. J. Greffet, and Y. De Wilde, Phys. Rev. Lett. 110, 146103 (2013).
[43] S. Dai, Z. Fei, Q. Ma, A. S. Rodin, M. Wagner, A. S. McLeod, M. K. Liu, W. Gannett, W. Regan, K. Watanabe, T. Taniguchi, M. Thiemens, G. Dominguez, A. H. Castro Neto, A. Zettl, F. Keilmann, P. Jarillo-Herrero, M. M. Fogler, and D. N. Basov, Science 343, 1125 (2014).

[44] A. Alu and N. Engheta, Phys. Rev. B 79, 235412 (2009).

[45] S. Campione, S. Steshenko, and F. Capolino, Opt. Express 19, 18345 (2011)

[46] A. Moroz, J. Opt. Soc. Am. B 26, 517 (2009).

[47] L. Lewin, Polylogarithms and Associated Functions (Elsevier, New York, 1981).

[48] R. Siegel and J. Howell, Thermal Radiation Heat Transfer (Taylor and Francis, New York, 2001).

[49] G. Chen, Nanoscale Energy Transport and Conversion: A Parallel Treatment of Electrons, Molecules, Phonons, and Photons (Oxford University Press, New York, 2005).

[50] E. D. Palik, Handbook of Optical Constants of Solids (Academic Press, Orlando, 1985).

[51] Y. Chalopin, H. Dammak, M. Hayoun, M. Besbes, and J. J. Greffet, Appl. Phys. Lett. 100, 241904 (2012). 